\title{
Estudo epidemiológico de pacientes portadores de infecção do complexo bucomaxilofacial tratados no Hospital Universitário de Maringá: estudo retrospectivo ao longo de 8 anos
}

\section{Epidemiological study of patients with oral maxillofacial infection treated at Universitary Hospital of Maringá: retrospective study about 8 years}

Gustavo Jacobucci Farah* José Henrique Santana Quinto** Izabella Giannasi Farah ${ }^{* * *}$ Marcelly Tupan Chistoffoli****

Caroline Resquetti Luppi ${ }^{* * * *}$

\section{Resumo}

Infecções agudas bucomaxilofaciais podem ser condições clínicas graves e de ocorrência comum, caracterizadas pela disseminação do processo infeccioso a tecidos adjacentes e espaços faciais da região de cabeça e pescoço, podendo resultar em várias complicações, até mesmo em óbito, embora seja raro. Objetivo: realizar uma análise epidemiológica de infecções maxilofaciais, relacionando os dados ao tratamento instituído e à sua efetividade, bem como analisar dados referentes a idade, sexo, principais dentes envolvidos e tempo total de internação. Sujeito e método: foram analisados retrospectivamente 240 prontuários de pacientes admitidos no Hospital Universitário de Maringá com infecção odontogênica, atendidos pela equipe de Cirurgia e Traumatologia Bucomaxilofacial no período de janeiro de 2009 a janeiro de 2017. Resultados: a média de idade dos pacientes foi de 38 anos, com 57 mulheres e 54 homens. A média de temperatura de admissão foi $38,5^{\circ} \mathrm{C}$. A região mais acometida foi o ramo posterior da mandíbula, tendo uma média de duração de infecção e hospitalização de 6,1 dias. A principal conduta foi drenagem e antibioticoterapia, sendo que cerca de 13 pacientes não precisaram desse tipo de intervenção, e um paciente evoluiu a óbito. Conclusão: com base nestes resultados e na literatura, infecções odontogênicas merecem atenção, pois podem ser fatais e requerem in- ternação rápida e tratamento adequado. Esse, portanto, é um assunto de grande importância para o cirurgião-dentista, que exerce papel fundamental na prevenção e no tratamento. A resolução precoce ainda é a forma mais adequada para evitar complicações mais graves.

Palavras-chave: Análise epidemiológica. Infecções bucomaxilofaciais. Infecções odontogênicas.

\section{Introdução}

Infecções maxilofaciais graves são caracterizadas pela disseminação do processo infeccioso a tecidos adjacentes e espaços faciais da região de cabeça, pescoço e tórax. Sua principal origem é odontogênica, resultante da infecção periapical e de doença periodontal. Outras causas incluem lacerações, fraturas, injeção anestésica e situações pós-cirúrgicas. ${ }^{1}$

Embora a maioria dos processos infecciosos em estágios iniciais seja bem controlada com intervenção cirúrgica e antibioticoterapia, alguns processos têm potencial para se disseminar pelos planos faciais da cabeça e do pescoço e acometer estruturas como órbita, seio cavernoso e mediastino, podendo causar o comprometimento de vias aéreas, resultando

\footnotetext{
Doutor, professor de Cirurgia e Traumatologia Bucomaxilofacial, Departamento de Odontologia, Universidade Estadual de Maringá, Maringá, PR, Brasil. Residente de Cirurgia e Traumatologia Bucomaxilofacial, Departamento de Odontologia, Universidade Estadual de Maringá, Maringá, PR, Brasil. Acadêmica de Odontologia, Universidade Estadual de Maringá, Maringá, PR, Brasil. Acadêmica de Odontologia, Universidade Estadual de Maringá, Maringá, PR, Brasil.
Acadêmica de Odontologia, Universidade Estadual de Maringá, Maringá, PR, Brasil.

Cirurgiã-dentista formada pelo Departamento de Odontologia da Universidade Estadual de Maringá, Maringá, PR, Brasil.
} 
em septicemia e morte..$^{2-6}$ Fatores como demora na procura por atendimento especializado, antibioticoterapia inicial inadequada, condições sistêmicas imunossupressoras e virulência do microorganismo podem contribuir para a disseminação rápida do processo infeccioso. ${ }^{2,5}$ Sendo assim, o conhecimento de condições que favorecem a progressão da doença é necessário ao profissional, com a finalidade de diagnosticar um quadro com gravidade potencial, sendo o cirurgião-dentista peça-chave em diagnóstico e tratamento desse tipo de patologia.

\section{Sujeitos e método}

O presente trabalho consistiu em um estudo observacional e transversal. Foram analisados 240 prontuários, dos quais 112 fizeram parte do estudo retrospectivo de pacientes com diagnóstico de infecção odontogênica, tratados pela equipe de Cirurgia e Traumatologia Bucomaxilofacial da Universidade Estadual de Maringá, no período de janeiro de 2009 a janeiro de 2017. A análise dos prontuários foi realizada com auxílio de um formulário estruturado, considerando as variáveis gênero, idade, principais dentes envolvidos, tempo total de internação, temperatura de admissão, condição sistêmica do paciente e tratamento instituído, e foi feita em ambiente apropriado do Departamento de Odontologia da Universidade Estadual de Maringá e do Hospital Universitário de Maringá. O protocolo do estudo e a isenção do termo de consentimento livre e esclarecido foram aprovados pelo Comitê de Ética em Pesquisa em Seres Humanos da Universidade Estadual de Maringá sob o parecer $n^{\circ}{ }^{\circ}$ 2.144.580.
Foram excluídos os prontuários que apresentavam preenchimento inadequado ou insuficiente, assim como não assinatura do termo de consentimento livre e esclarecido. Os dados coletados foram transferidos para planilha do Microsoft Office Excel® 2013 (Microsoft Corporation@, EUA), para organização tabular, e o processamento das informações registradas ocorreu por estatísticas descritiva e analítica.

\section{Resultados}

Dos 112 pacientes analisados, 57 eram do sexo feminino (51\%) e 54 do sexo masculino (49\%), com idade média de 38 anos. Desses, 2,67\% chegaram ao hospital com febre (acima de $37,5^{\circ} \mathrm{C}$ ); 15,17\%, em estado febril (37ㅇ a $\left.37,5^{\circ} \mathrm{C}\right)$; e $75 \%$, em estado afebril (abaixo de $37^{\circ} \mathrm{C}$ ). A região mais acometida foi o ramo posterior da mandíbula, incidindo mais pelo lado esquerdo, sendo os molares inferiores os mais acometidos. Obteve-se uma média de duração da infecção e da hospitalização de 6,1 dias, e a maioria dos pacientes $(81,25 \%)$ não apresentava nenhuma doença sistêmica concomitante. Em relação ao tratamento, o principal foi drenagem com antibioticoterapia $(48,2 \%)$, sendo que 13 pacientes $(11,6 \%)$ não precisaram desse tipo de intervenção, conforme a Figura 1, e somente um $(0,9 \%)$ evoluiu a óbito.

Os antimicrobianos mais utilizados foram: Unasyn $^{\circledR} 3 \mathrm{~g}$ (ampicilina + sulbactam), em 23,2\% dos tratamentos; Unasyn ${ }^{\circledR} 1,5 \mathrm{~g}$, em $22,3 \%$; Flagyl ${ }^{\circledR}$ $500 \mathrm{mg}$ (metronidazol), em 16\%; e Keflin ${ }^{\circledR} 1 \mathrm{~g}$ (cefalotina), em 10,7\%, conforme o Quadro 1.

Figura 1 - Tratamento utilizado nos pacientes internados com infecções odontogênicas

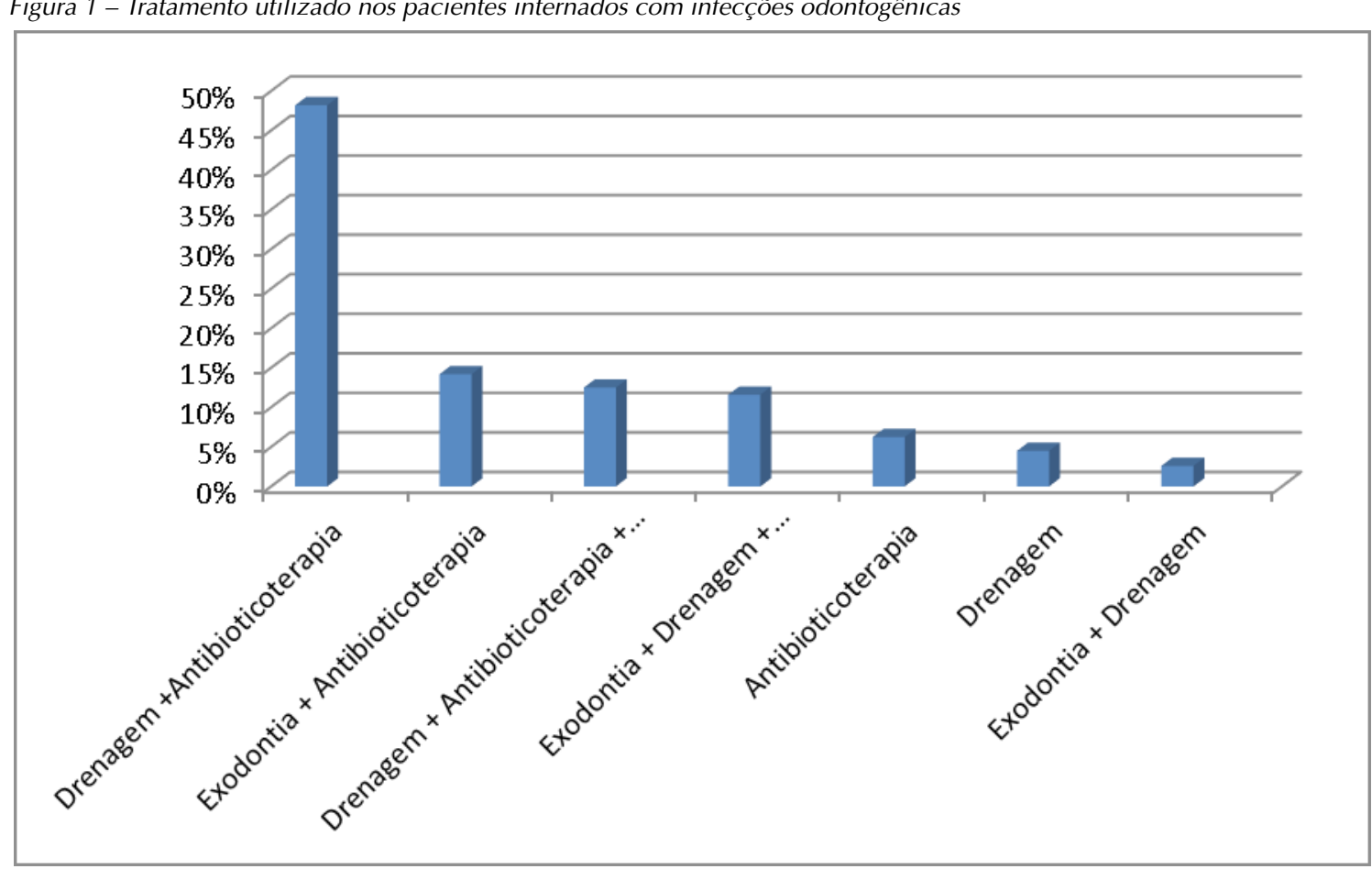

Fonte: autores. 
Quadro 1 - Porcentagem dos antimicrobianos utilizados no tratamento das infecções odontogênicas

ANTIMICROBIANOS

\begin{tabular}{|l|c|}
\hline AMPICILINA + SULBACTAM 3g & $23,2 \%$ \\
AMPICILINA + SULBACTAM 1,5g & $22,3 \%$ \\
METRONIDAZOL 500mg & $16 \%$ \\
CEFALOTINA 1g & $10,7 \%$ \\
\hline
\end{tabular}

Fonte: autores.

\section{Discussão}

As infecções maxilofaciais representam uma área relevante do conhecimento médico, pois podem evoluir para quadros de alta morbidade e mortalidade. Exigem, portanto, plena capacitação profissional tanto na prevenção quanto no diagnóstico e, sobretudo, na sua resolução clínica. ${ }^{7}$ É importante conhecer sua epidemiologia, para desenvolver estratégias de prevenção e elaborar o tratamento. ${ }^{8}$ Nesta pesquisa, foi desenvolvido um estudo retrospectivo, por meio da análise de 112 prontuários de pacientes portadores de infecção de origem odontogênica. As características clínicas dos pacientes encontradas nos registros dos prontuários indicaram processo infeccioso mais grave, necessitando de admissão hospitalar, assim como mostram outros estudos na área. Esse tipo de infecção, embora apresente predileção pelo gênero masculino, ${ }^{9}$ nesta pesquisa, houve uma diferença insignificante entre os gêneros, e o gênero feminino prevaleceu. Entretanto, diversos estudos observam que podem ocorrer essas variações, devido à diferença demográfica. ${ }^{1,10}$ Normalmente, originam-se da região posterior da mandíbula de jovens adultos, ${ }^{9,11}$ sendo a idade com maior frequência entre 20 e 29 anos,,$^{12}$ no entanto, nesta pesquisa, a média de idade foi de 38 anos.

A maioria dos autores atribui o espaço submandibular à maior frequência do acometimento. ${ }^{10,11,13}$ Este trabalho ratifica essa observação literária, uma vez que a maioria da amostra apresentou envolvimento do espaço submandibular.

O período médio de internação foi de 6,1 dias, semelhante ao descrito por outros autores. ${ }^{14,15} \mathrm{O}$ protocolo de tratamento baseou-se em antibioticoterapia associada, quando necessário, à drenagem cirúrgica. Essa conduta está de acordo com outros trabalhos que indicam, nos casos em que após a terapia medicamentosa não se observa melhora do quadro, a cirurgia de drenagem do espaço comprometido. ${ }^{8,16}$

Foi observado, nos registros, o uso frequente de ampicilina e sulbactam. Esse antibiótico é indicado para casos em que há suspeita da presença de microrganismos resistentes aos antibióticos $\square$-lactâmicos; é um medicamento bem tolerado e seguro para a terapia empírica de infecções de origem odontogênica, uma vez que é eficiente contra as bactérias aeróbicas e anaeróbicas responsáveis pelo quadro infeccioso. ${ }^{6}$

\section{Conclusão}

Com base nos resultados encontrados e na revisão de literatura, conclui-se que:

1. houve predomínio de infecções maxilofaciais em adultos jovens, afetando ambos os sexos com praticamente a mesma frequência;

2. as infecções usualmente envolvem mais de um espaço facial, sendo o submandibular o mais frequente;

3. o protocolo de tratamento mais encontrado é composto de drenagem cirúrgica precoce, antibioticoterapia endovenosa e remoção da causa.

Portanto, este é um assunto de grande importância para o cirurgião-dentista, que exerce papel fundamental na prevenção e no tratamento adequado, sendo a resolução precoce ainda é a forma mais adequada de se evitar possíveis complicações de grave potencial.

\section{Abstract}

Acute Oral maxillofacial infections can be serious and relatively common clinical conditions, characterized by the spread of the infectious process to adjacent tissues and facial spaces of the head and neck region, which can result in several complications and lead to even death, although it is rare. Objective: the objective of this study was to perform an epidemiological analysis of maxillofacial infections and relate their data to the treatment instituted and the effectiveness of the same, as well as to analyze data regarding the age, sex, main teeth involved and total time of hospitalization. Subjects and method: in order to carry out study, 240 medical recordswere analyzed retrospectivelyat the University Hospital of Maringá of the patients with odontogenic infection attended by the Oral Maxillofacial Surgeon in the period of January 2009 to January 2017. Results: as a result, mean age was 38 years, with 57 women and 54 men and mean intake temperature was $38.5 \square$. The most affected region was the posterior branch of the mandible, with a mean duration of infection and hospitalization of 6.1 days. The main conduct was drainage and antibiotic therapy, and about 13 patients did not need this intervention and only 1 died. Conclusion: Based on these results and in literature, attention should be paid to odontogenic infections, which can be fatal and require proper treatment. This is a subject of great importance for the dentist, who plays a key role in prevention and proper treatment, its early resolution is still the most appropriate way to avoid serious complications.

Keywords: Epidemiological analysis. Oral maxillofacial infections. Odontogenic infections. 


\section{Referências}

1. Wang J, Ahani A, Pogrel MA. A five-year retrospective study of odontogenic maxillofacial infections in a large urban public hospital. Int J Oral Maxillofac Surg 2005; 6:646-9.

2. Bullock JD, Fleishman JA. The spread of odontogenic infections to the orbit: diagnosis and management. J Oral Maxillofac Surg 1985; 43:749-55.

3. Cai XY, Zhang WJ, Zhang ZY, Yang C, Zhou LN, Chen ZM. Cervical infection with descending mediastinitis: a review of six cases. Int J Oral MaxillofacSurg 2006; 35:1021-5.

4. Kim I, Kim J, Jang K, Moon Y, Park S. Orbital abscess from an odontogenic origin. Oral Surg Oral Med Oral Pathol Oral Radiol Endod 2007; 103:e1-e6.

5. Ogundiya DA, Keith DA, Mirowski J. Cavernous sinus thrombosis and blindness as complications of an odontogenic infection: report of a case and review of the literature. J Oral Maxillofac Surg 1989; 47:1317-21.

6. Larawin V, Naipao J, Dubey SP. Head and neck infections. Otolaryngol Head Neck Surg 2006; 135:889-93.

7. Lian HT, Tsai CS, Chen YL, Liang JG. Influence of diabetes mellitus on deep neck infection. J Laryngol Otol 2006; 8:650-4.

8. Peterson LJ, Ellis IIIE, Tucker MR. Cirurgia Oral e Maxilofacial Contemporânea. 4. ed. Rio de Janeiro: Guanabara koogan; 2003.

9. Dodson TB, Barton JA, Kaban LB. Predictors of outcome in children hospitalized with maxiloffacial infections: a linear logistic model. J Oral Maxillofac Surg 1991; 49:838-42.

10. Flynn TR, Shanti RM, Levi MH. Severe odontogenic infections, part 1: prospective report. J Oral Maxillofac Surg 2006; 7:1093-103.
11. Flynn TR, Shanti RM, Hayes C. Severe Odontogenic Infections, Part 2: Prospective Outcomes Study. J Oral Maxillofac Surg 2006; 64(7):1104-13.

12. Kannangara DW, Thadepalli H, Mcquirter JL. Bacteriology and treatment of dental infections. Oral Surg Oral Med Oral Pathol 1980; 2:103-9.

13. Ariji Y, Gotoh M, Kimura Y, Naitoh K, Kurita K, Natsume $\mathrm{N}$. Odontogenic infection pathway to the submandibular space: imaging assessment. Int J Oral Maxillofac Surg 2002; 31:165-9.

14. Sánchez R, Mirada E, Arias J. Severeodontogenicinfections: Epidemiological, microbiologicalandtherapeuticfactors. Med Oral Patol Oral Cir Bucal 2011; 16(5):670-6.

15. Sette-Dias AC, Maldonado AJ, Aguiar EG. Profile of patients hospitalized with odontogenic infections in a public hospital in Belo Horizonte. Brazil. J Cln Exp Dent 2012; 4:271-4.

16. Krishnan V, Johnson JV, Helfrick JF. Managemento of maxillofacial infections: a review of 50 cases. J Oral Maxillofac Surg $1991 ; 51: 868$.

Endereço para correspondência:

José Henrique Santana Quinto

Avenida Mandacaru, 1550, Centro

87080-000, Maringá, Paraná, Brasi

Telefone: (44) 3011-9055

E-mail: zesantanaquinto@gmail.com

Recebido: 30/08/18. Aceito: 04/10/18. 